\title{
Método científico. El papel del investigador y su responsabilidad en Leben des Galilei de Bertolt Brecht: Estudio analítico e interpretativo
}

\author{
José Alberto Sánchez Berbegal \\ Universidad de Sevilla \\ alberto.sanchez.berbegal@gmail.com \\ https://dx.doi.org.10.12795/futhark.2019.il4.14
}

Fecha de recepción: 20.03 .2018

Fecha de aceptación: 1.05.2019

Resumen: El presente estudio constituye un ejercicio de recopilación de los principales aspectos que atañen al método científico y al papel del investigador y su responsabilidad en la obra Leben des Galilei de Bertolt Brecht. El núcleo del análisis se centra principalmente en la figura del protagonista, el científico italiano Galileo Galilei. En la actualidad, al igual que en el pasado, los científicos juegan un papel fundamental en la sociedad. Por este motivo, se antoja necesario conocer las responsabilidades que recaen sobre este colectivo, de manera que la humanidad sea consciente de la imperiosa necesidad de austeridad en este campo. El pasado siglo $X X$, marcado por encarnizadas guerras, supuso un enorme desarrollo para la tecnología. Pero, ¿con qué finalidad se desarrolló esta tecnología? Lo cierto es que el interés por su desarrollo estuvo marcado por numerosos motivos bélicos. Muchos fueron los científicos que pusieron sus conocimientos al servicio del poder sin llegar a pensar que estos se utilizarían para tales fines. El propio Albert Einstein, defensor del desarrollo de la bomba atómica, se arrepintió de ello tras cerciorarse del uso que se había hecho de ella en territorio nipón.

Palabras clave: Responsabilidad científica, Método científico, Desarrollo del conocimiento.

Scientific Method. The Role of the Researcher and his Responsibility in Bertolt Brecht's Leben des Galilei: Analytical and Interpretative Study 
Abstract: This study represents a compilation of the main aspects regarding the scientific method and the role of the researcher and his responsibility in Bertolt Brecht's drama Leben des Galilei. The focus of the analysis is set mainly on the figure of the protagonist, the italian scientist Galileo Galilei. Nowadays, as well as in the past, scientist have a crucial role in society. For this reason, it seems essential to know the responsibilities of this collective, so that humanity becomes conscious of the imperative need for austerity in this field. The 20th century, characterized by savage wars, represents an immense development regarding technology. Which purpose had this development? The motivation was actually influenced by numerous warlike reasons. Many scientists put their knowledge at the service of power without even considering that they would be used for such purposes. Albert Einstein, defender of the development of the atomic bomb, regretted this after having ascertained the use that had been made of it in Japan.

Keywords: Scientific responsibility, Scientific method, Development of knowledge.

Sumario: I. Responsabilidad cientifica. Leben des Galilei y Bertolt Brecht. 2. La responsabilidad de Galileo y su método científico. 3. Símbolos. 3.I. Ceguera y visión. 3.2. Comida. 3.3. Vestimenta. Conclusiones

\section{Responsabilidad científica. Leben des Galilei y Bertolt Brecht}

El papel de los científicos a lo largo de la historia ha resultado fundamental para el desarrollo colectivo e intelectual de las civilizaciones. La curiosidad es algo intrínseco al ser humano, como se puede observar en las primitivas manifestaciones artísticas localizadas en cuevas o en las herramientas encontradas pertenecientes a seres humanos nómadas de la Edad de Piedra. El hombre tiende a cuestionarse a sí mismo, a establecer hipótesis $y$, finalmente, a contrastarlas. Cuando la falta de medios impide corroborar las tesis, es frecuente la aparición de creencias para suplir dicha carencia.

Los cientíicos, como perseguidores de la verdad, tienen una gran responsabilidad para con la sociedad. Los investigadores se han encontrado habitualmente con un problema de financiación. Para poder desarrollar investigaciones es necesario contar con una seguridad, proporcionada por el tiempo, la libertad' y la financiación; es decir, un mecenazgo:

Los mecenazgos constituían redes sociales en torno a los príncipes y a los aristócratas. Facilitaban la comunicación entre científicos, enmarcando su

I "El conocimiento científico implica libertad de pensamiento y comunicación” (Zanarini 2017: 49I). 
identidad socioprofesional, les otorgaban estatus y legitimidad social (Zanarini 2017: 494).

Esto causa una limitación. Quien no se encontrara dentro de estas redes sociales, no podía investigar. Además, la dependencia económica de un mecenas podía provocar que este pudiera pedir al científico alguna compensación.

A. Weinberg establece una condición indispensable que se debe cumplir en un científico con independencia de sus características y habilidades, la responsabilidad: "A scientist can be brilliant, imaginative, clever with his hands, profound, broad, narrow - but he is not much as a scientist unless he is responsible" (Weinberg 1978: I). Además, se establecen dos tipos de responsabilidad. Por una parte aparece la responsabilidad de comunicar los resultados: "associated with basic research and the communication of the results" (Edsall 198I: II). Por otra parte se establece la responsabilidad social: "such matters as the control of nuclear and other weapons, the uses and hazards of toxic chemicals and radioactive materials" 2 . Por tanto, los científicos deben ser responsables y estar comprometidos con la sociedad y el conocimiento. "the empirical sciences have been shaped by bitter social struggles" (Suvin 1990: 128). El contexto histórico bélico ${ }^{3}$ con el que cuentan las ciencias empíricas podría estar determinado por los mecenazgos y la falta de responsabilidad.

La obra Leben des Galilei ${ }^{4}$ de Bertolt Brecht fue publicada en el año 1939 en el exilio danés. El protagonista es el científico italiano Galileo Galilei ${ }^{5}$, fiel defensor de la teoría heliocéntrica. En la obra se tematiza la lucha de Galileo contra el poder establecido, la Iglesia. Hay un conflicto de intereses que hace que el protagonista claudique y decida dejar de lado su responsabilidad para con la verdad empírica. Este sentido de responsabilidad del científico aparece en la obra y se puede

\footnotetext{
2 lbíd.

${ }^{3}$ En la actualidad se tiene en cuenta el contexto histórico en la metodología de la enseñanza de la física y otras ciencias empíricas. "Al plantear un modelo físico como la solución a un problema, se evita incurrir en el error epistemológico de mostrarles a los alumnos un empirismo ingenuo, que introduce la recolección de datos y la generación de hipótesis sin mencionar el problema histórico que generó la investigación, sin tener en cuenta las distintas soluciones que se plantearon -muchas de ellas erróneaspara abordar el problema, ignorando las tensiones entre los intereses de los científicos y las disputas políticas y sociales en torno a sus investigaciones" (Zanarini 2017: 492).

4 "Het Galilei-stuk is zijn meest autobiografische toneeltekst. Anders geformuleerd - het is de toneeltekst waar brokstukken van zijn biografie in terecht zijn gekomen” (Riemen 20I I: 106).

5 "Los resultados de sus investigaciones en áreas como la cinemática y la astronomía sentaron las bases conceptuales de la nueva física” (Zanarini 2017: 49I).
} 
relacionar con otro personaje histórico, Albert Einstein ${ }^{6}$. Este célebre científico, defensor del desarrollo de la bomba atómica, mostró su arrepentimiento cuando contempló el uso que se hizo de ella en territorio japonés ${ }^{7}$ :

it was this sense of responsibility which led Einstein to write a letter urging President Roosevelt to inaugurate a program to develop the atomic bomb. ... Einstein later felt a much heavier responsibility for its unfortunate success (Dorsey 1987: 279).

Como afirma P. R. Paulsell, la Segunda Revolución Científica, marcada por Einstein, influenció a Brecht en la escritura de esta obra: "the impact of the second 'Scientific Revolution' ushered in by Einstein and the reevaluation by contemporary historians of science of the role of Galileo in the first 'Scientific Revolution"' (Pausell 1988: 267). Así como también lo hizo el papel de Galileo en la Primera Revolución Científica.

Bertolt Brecht toma la historia como didáctica ${ }^{8}$ :"Brecht is assuming, that the function of history is didactic: we have both a modern preoccupation with authenticity and a desire to use the past to point morals for the present" (Cohen 1970: 8I). Esto encaja con el objetivo del dramaturgo y de su teatro épico de inducir a los espectadores a una reflexión que posteriormente les lleve a actuar para conseguir un cambio en la sociedad. En todos los ámbitos, incluido el de las ciencias empíricas, la historia se repite. Mostrando unos hechos históricos situados

\footnotetext{
${ }^{6}$ Es conocido que Brecht fue influenciado por las teorías de Einstein, algo común en las décadas de los años 20 y 30 del siglo XX: "Since Brecht is known to have used popularizations of Einstein's theories as sources for his play and since Einstein's discoveries were topics of even parlor conversations all over the world during the 1920s and 1930s, it seems reasonable to assume that Brecht was influenced both implicity and explicity by Einstein's discoveries" (Pausell 1988: 273).

7 "the explosion of the atomic bomb in Hiroshima caused Brecht to change his opinion of the significance of this betrayal and to make several alterations in the play" (Fehn 1978: 32). Tras esto, Brecht publicó una segunda obra: "Nadat Brecht in juli 194I met zijn familie in de Verenigde Staten is aangekomen, werkt hij aan plannen om Leben des Galilei in Amerika opgevoerd te krijgen. Vanaf 1944 wordt hij daarin gesteund door de van oorsprong Engelse filmacteur Charles Laughton“" (Riemen 201 I: 114).

${ }^{8}$ Existe un estudio que experimentó la utilidad del teatro científico para la enseñanza de la ciencia. Si bien en la física cada vez se recurre más al contexto histórico, parece ser que el teatro es una herramienta francamente productiva que permite establecer debates en torno a él: "Através do diálogo, instigamos os alunos a entender que sendo o conhecimento uma construção humana ele depende de como nós o construímos e pode estar errado. Esta discussão permeou os nossos estudos sobre a Revolução Copernicana. Quais eram os interesses daqueles que defendiam o Geocentrismo, e porque se sentiam ameaçados pelo Heliocentrismo a ponto de punir seus defensores severamente?" (Gimenez 2015: 82).
}

\section{2}


en Italia consigue Brecht ${ }^{9}$ que el espectador reflexione sobre la situación reinante en su momento histórico, pero sin llegar a la identificación propia del teatro aristotélico. Brecht trata de evitar esta identificación para conseguir que la sociedad reflexione de manera individual y sea crítica; de lo contrario, se limitarían simplemente a imitar lo que ven representado en la escena ${ }^{10}$.

\section{La responsabilidad de Galileo y su método científico}

La obra tematiza la vida del científico italiano Galileo Galilei durante un periodo de 28 años. Galileo, defensor de la teoría heliocéntrica, lleva a cabo investigaciones astronómicas cuyos objetivos son demostrar este hecho. Por su parte, la Iglesia, que ostenta un gran poder dentro del estado, se mantiene reacia a aceptar sus investigaciones por ir en contra de los intereses de la propia institución. Esto provoca que en la obra se puedan reconocer dos temas: "'the endangered knower' and 'the betrayal of science"' (Suvin 1990: 127). Galileo corre peligro al continuar con sus investigaciones, lo que provoca que se retracte $y$ las abandone".

El drama comienza en el pobre gabinete de trabajo de Galileo. Tanto el entorno de trabajo como la situación de Galileo, incapaz de pagar al lechero, manifiestan la precariedad en la que se encontraba el científico. Sin embargo, este emplea su dinero en comprar libros e investigar. Las autoridades venecianas aportan un sustento mensual a Galileo, pero este no es suficiente. Por ello, se ve en la necesidad de aceptar alumnos privados. Con esto se demuestra que "La universidad era para la República de Venecia una empresa que producía ganancias" (Zanarini 2017: 494), puesto que aunque la institución era consciente del gran potencial investigador de Galileo, no posibilitaba que este se pudiera dedicar en exclusiva a las investigaciones. Solo se podía recibir por las ciencias el dinero que

\footnotetext{
${ }^{9}$ Bertolt Brecht escribe un drama cuyo protagonista es un científico. El hecho de que Brecht sea un dramaturgo podría haber influido en el tratamiento que se hace de la perspectiva del científico en esta obra: "A maioria das peças que abordam a ciência não é escrita por cientistas, mas por dramaturgos com manifestos conhecimentos em teatro, ao exemplo de Bertolt Brecht (Alemanha) e Osvaldo Mendes (Brasil). Talvez por essa razão, estes geralmente abordam o tema na perspectiva do cientista e não somente das ideias científicas" (Braga / Medina 2010: 317).

${ }^{10}$ Situar la escena en el pasado y en un lugar en el que no residen los espectadores a los que Brecht quiere hacer reflexionar es un recurso que emplea el autor en sus obras pertenecientes al teatro épico. Con estos recursos consigue crear el Verfremdungseffekt.

1 I Oficialmente, tras el requerimiento de la Iglesia y la Inquisición, abandona sus investigaciones. Sin embargo, lo cierto es que finalmente, con la entrega de Discorsi, demuestra que no dejó completamente de lado su implicación con la ciencia. Pese a ello, Galileo consideraba que había fallado como científico.
} 
estas generaran: "Sie können für das Wissen, das Sie verkaufen, nur so viel verlangen, als es dem, der es Ihnen abkauft, einbringt” (Brecht 2017: 18) ${ }^{12}$. El propio secretario de la universidad pide a Galileo que continúe produciendo inventos que ayuden en el ámbito militar: "machen Sie doch mal wieder was so Hübsches wie Ihren famosen Proportionalzirkel, ... und die Schwere von Kanonenkugeln bestimmen kann" (19-20). Con esto se manifiesta que lo importante es poner el conocimiento al servicio del poder; sin embargo, no se tiene en cuenta la necesidad de saber que tiene la población, la cual Galileo quiere satisfacer.

El protagonista asegura a su discípulo Andrea que se encuentran en el comienzo de una nueva época: "die alte Zeit ist herum, und es ist eine neue Zeit" (8). A esto llega Galileo a través de sus observaciones ${ }^{13}$ de los astros. Su objetivo es llevar el nuevo conocimiento científico a toda la población, tarea que se le hace más sencilla cuando recibe un artilugio nuevo procedente de Holanda. Se trata de un telescopio, el cual Galileo mejora y emplea para observar con exactitud el movimiento de algunos cuerpos en el espacio. Gracias a estas observaciones, Galileo asevera sus hipótesis y afirma "Himmel abgeschafft" (28). Además, el telescopio sirve para que el científico reciba, mediante su entrega, más dinero por parte de las autoridades: "Galileo has used his telescope not only to trick the city fathers of Padua into giving him more money, but also to prove the Copernican theory" (Fehn 1978: 28). Su precariedad lo motiva a realizar esta acción de apropiarse de un invento ajeno. Solo así podrá seguir con sus investigaciones. Cuando entrega el supuesto nuevo invento, las autoridades lo conciben con una utilidad bélica:

\begin{abstract}
Und ist es Ihnen beigefallen, daß wir vermittels dieses Instruments im Kriege die Schiffe des Feinds nach Zahl und Art volle zwei Stunden früher erkennen werden als die unsern, so daß wir, seine Stärke wissend, uns zur Verfolgung, zum Kampf oder zur Flucht zu entscheiden vermögen? (24).
\end{abstract}

Galileo, que empleó el telescopio para observar la luna, solo lo concibe como un elemento útil para el desarrollo científico y del conocimiento, no como un elemento para utilizar en la guerra: "Ich kann dir nicht versprechen, daß ich den Karneval hier durchstehen werde. Die meinen hier, sie kriegen einen einträglichen Schnickschnack, aber es ist viel mehr. Ich habe das Rohr gestern nacht auf den Mond gerichtet" (24).

\footnotetext{
${ }^{12}$ Todas las referencias son de esta edición.

13 "The ancients, Einstein stressed, tended to devalue the importance of observation of the physical world" (Paulsell 1988: 270). Sin embargo, Galileo basa sus investigaciones y sus afirmaciones en la observación.
} 
La responsabilidad imperante en la figura de Galileo es tan grande que sus investigaciones continúan: "Der Mond kann eine Erde sein mit Bergen und Tälern, und die Erde kann ein Stern sein. Ein gewöhnlicher Himmelskörper, einer unter Tausenden" (28). Tras el descubrimiento de montañas y valles en la luna, compara este astro con la tierra. Relativiza la importancia de la tierra, relegando su posición en el universo a la de cualquier otro cuerpo celeste. Este descubrimiento, junto con otras afirmaciones anteriores como la abolición del cielo (Himmel abgeschafft ${ }^{14}$ ) - la validez de la teoría heliocéntrica, suponen un problema para Galileo. La Iglesia no va a permitir que esas ideas se difundan entre la población, ya que, como escucha Galileo de un miembro de la Iglesia en una conversación privada entre ambos, van en contra de los intereses de la propia institución eclesiástica ${ }^{15}$. Sin embargo, el científico italiano cree en las personas y su necesidad innata de conocimiento: "Ja, ich glaube an die sanfte Gewalt der Vernunft über die Menschen. Sie können ihr auf die Dauer nicht widerstehen. ... Das Denken gehört zu den größten Vergnügungen der menschlichen Rasse" (34-35). "he believes in them as the future standard-bearers of science - astronomy will be talked about in the marketplaces and will be welcomed there" (Cohen 1970: 86). Asimismo, Galileo alega que esas personas son las que mantienen su motivación y su fuerza: "Ohne diesen Glauben würde ich nicht die Kraft haben, am Morgen aus meinem Bett aufzustehen" (34). Por consiguiente, el propósito de Galileo es extender no solo el conocimiento entre la población, sino también la duda: "The drama's Galileo wants more than to spread scientific knowledge to the people. He also wants to spread the spirit of doubt" ${ }^{\prime 6}$ (Cohen 1970: 88). Esto significa que Galileo busca provocar la reflexión, al igual que busca Bertolt Brecht con el teatro épico.

La utopía que presenta Galileo en la obra se basa en la razón crítica. Mediante ella, el protagonista considera que podrá continuar con sus investigaciones, expandir el conocimiento científico y no ser juzgado por la Iglesia y la Inquisición: "Galileo's utopia will essentially consist of the use of critical reason to think against the power of institutionalized reason" (Sohlich 1993: 5I). Por ello, a pesar de la insistencia de su amigo Sagredo por evitarlo, decide ir a Florencia para

\footnotetext{
${ }^{14}$ Citado con anterioridad; (Brecht 2017: 28).

${ }^{15}$ F. S. Taylor manifiesta que la sociedad de la época de Galileo era corrupta y sin moral. Esto fue la causa de los numerosos problemas derivados de sus investigaciones: "It must not be thought that Galileo, born in the same year as Shakespeare, was born into the same brave world of expanding knowledge, wealth and genius. Rather did he encounter a corrupt and sterile society, whose influence may account for much of what we might wish to see expunged from his record. ... The morals of Machiavelli and the cheerfully avowed villainies of Benvenuto Cellini may afford a measure of the tone of sixteenth century Italy. ... Such, then, was the background of a scientific man in the late sixteenth century - an expanding world, a repressive Church, and a corrupt society" (Taylor 1938: 7-8).

16 "doubt appears to be the central feature of scientific thinking for Brecht" (Cohen 1970: 88).
} 
exponer sus tesis: "Ich werde sie bei den Köpfen nehmen und sie vor das Rohr schleifen. Auch die Mönche sind Menschen" (38). Galileo entiende que si los monjes son personas, deberían rendirse antes las evidencias y dejarse llevar por los instintos humanos que se acercan al conocimiento. Sagredo tilda finalmente de crédulo a su amigo: "bist du leichtgläubig wie ein Kind in allem"17 (39). Pese a que las autoridades rehúsan de la posibilidad que les brinda Galileo de mirar por su telescopio, este afirma "Ich würde meinen, als Wissenschaftler haben wir uns nicht zu fragen, wohin die Wahrheit uns führen mag" (49). A pesar de dicho rechazo, Galileo sigue comprometido; incluso rechaza abandonar su casa cuando hay una epidemia de peste, ya que no quiere perder sus libros y cuadernos.

Galileo recibe burlas por parte de miembros de la Iglesia. Algunos de ellos llegan a mostrar explícitamente que este científico debe ser tratado como un enemigo por apartar la tierra a un segundo lugar mediante sus teorías: "Ich höre, dieser Herr Galilei vesetzt (sic) den Menschen aus dem Mittelpunkt des Weltalls irgendwohin an den Rand. $\mathrm{Er}$ ist folglich deutlich ein Feind des Menschengeschlechts! Als solcher muß er behandelt werden" (6I). También se compara al protagonista de la obra con Giordano Bruno ${ }^{18}$ : "das sehe ich doch, daß Sie diesem Menschen, den wir seinerzeit verbrannt haben - wie hieß er doch? auffallend gleichen" (62). A pesar de todo, el astrónomo de la Iglesia confirma que las teorías son válidas. Sin embargo, dicha institución ignora el veredicto y prohíbe las tesis de Galileo. Por tanto, en este fragmento de la obra se tematiza la lucha entre la Iglesia y Galileo: "The play shows, on the pragmatic hand, two competing types of institutions, the Church/State Establishment and Galileo's troop, a budding counter-Establishment of science" (Suvin 1990: 126). A partir de este suceso, Galileo pasa ocho años sin investigar, no sin antes asegurar que "Wer die Wahrheit nicht weiß, der ist bloß ein Dummkopf. Aber wer sie weiß und sie eine Lüge nennt, der ist ein Verbrecher!"19 (8I).

\footnotetext{
17 “Die Naivität ist sowohl eine Eigenschaft der Greise wie auch der Kinder. Und der Mann ist, der das Kind und den Greis in sich enthält" (Brecht 1967: 44). Brecht trata en Schriften zur Literatur und Kunst la ingenuidad con la que Sagredo caracteriza a Galileo.

18 "La figura de Giordano Bruno aparece a lo largo de la obra de Brecht como una referencia al contexto histórico de la época, marcando las tensiones entre la ciencia nueva y el poder. Bruno publica libros considerados heréticos. Entre ellos se destaca: Del Infinito, El Universo y Los Mundos, donde expone una visión idiosincrática del copernicanismo y la filosofía natural. Refuta las nociones de Sagrada Trinidad y de Inmaculada Concepción, se opone a la ciencia aristotélica y propone la idea de que podría llegar a existir vida inteligente en otros planetas. Tras permanecer siete años preso en una cárcel de la Inquisición, donde es periódicamente torturado, el 17 de febrero de 1600 Giordano Bruno es ejecutado por orden del papa (sic) Clemente VIII" (Zanarini 2017: 495).

19 "Eine Hauptursache der Armut in den Wissenschaften ist meist eingebildeter Reichtum" (85).
} 
El periodo de ocho años en los que Galileo no continúa con sus investigaciones prohibidas por la Iglesia se fundamenta en el hecho de que su vida corre peligro: "Ich kann es mir auch nicht leisten, daß man mich über einem Holzfeuer röstet wie einen Schinken"20 (84). Con la llegada al poder de un nuevo Papa científico, Galileo retoma las investigaciones y se plantea dejar de escribir en latín para hacerlo en la lengua del pueblo, de manera que su ciencia sea más accesible para los ciudadanos. Durante diez años se difunden con éxito sus teorías, que llevan a causar una revolución popular; suponen una liberación con respecto a la Iglesia. Pero las esperanzas e investigaciones de Galileo no tienen más recorrido, puesto que la Iglesia lo interroga en Roma. El Inquisidor expone al Papa que con las teorías de Galileo ya no se necesita más la figura de Dios. Por ello, Galileo se ve en la obligación de retractarse públicamente. Esta retractación provoca el disgusto de sus amigos y discípulos, quienes pensaban que Galileo se mantendría firme y fiel ante sus teorías.

Andrea, discípulo de Galileo, visita a su maestro. Este ya es anciano y tiene serios problemas de visión. Este alumno, cuyo enfado por la retractación de su profesor fue mayúsculo, se asombra al conocer que Galileo, de manera clandestina tras su enmienda pública, ha llevado a cabo una intensa labor de recopilación de sus investigaciones sobre mecánica y leyes de gravitación. Todo está agrupado en un solo tomo, Discorsi. Galileo encomienda a Andrea la misión de llevar el libro fuera de Italia, de manera que sus conocimientos se expandan. Galileo se autodenomina traidor por su retractación: "it ends optimistically, with Galileo denouncing himself as having been a 'criminal,' and a 'traitor' to his fellow beings" (Merriam-Paskow 1992: 42). Menciona al vencedor, aclara las dificultades de llevar a cabo una investigación científica en solitario y transmite otro motivo de su retractación: "Sie sind die Sieger. Und es gibt kein wissenschaftliches Werk, das nur ein Mann schreiben kann. ... Ich habe widerrufen, weil ich den körperlichen Schmerz fürchtete" (123). Andrea se sorprende por lo que su maestro ha hecho:

Andrea, discovering that Galileo has tricked his guards and has secretly made a copy of the Discorsi, decides that what he believed to be a simple cowardice was instead a demonstration of a new profesional ethic, and apologizes profusely for having misjudged his former teacher (Fehn 1978: 31).

Finalmente expresa Galileo cuál es el fin de la ciencia:

Ich halte dafür, daß das einzige Ziel der Wissenschaft darin besteht, die Mühseligkeit der menschlichen Existenz zu erleichtern. Wenn Wissenschaftler, eingeschüchtert durch selbstsüchtige Machthaber, sich damit begnügen, Wissen um des Wissens willen aufzuhäufen, kann die Wissenschaft zum

${ }^{20}$ De nuevo se hace referencia a Giordano Bruno; esta vez de forma implícita. 
Krüppel gemacht werden, und eure neuen Maschinen mögen nur neue Drangsale bedeuten (125).

Debido a que él, como hombre de ciencia, por momentos dejó de lado la investigación por las amenazas del poder, manifiesta su arrepentimiento. De no ser por eso, según Galileo los científicos podrían haber producido un gran avance. Además, reconoce que nunca estuvo realmente en serio peligro: "Ich habe zudem die Überzeugung gewonnen, Sarti, daß ich niemals in wirklicher Gefahr schwebte. Einige Jahre lang war ich ebenso stark wie die Obrigkeit. Und ich überlieferte mein Wissen den Machthabern" (126). Igualmente, añade que una persona como él no debe tolerarse en el mundo de las ciencias: "Ein Mensch, der das tut, was ich getan habe, kann in den Reihen der Wissenschaftler nicht geduldet werden" (I26). La obra concluye con Andrea entregando Discorsi en la frontera. Galileo ha conseguido su cometido'. hedonista:

A lo largo de la obra, Galileo actúa siempre siguiendo un propósito

when the peculiar circumstances of his life split apart his over-lapping purposes and place him in the dilemma of having to choose between a humanitarian and a hedonistic purpose, he always chooses the latter, and with hardly any hesitation. The last and most extreme in a series of his betrayals of people is his recantation, under pressure (but not torture) from the ruling authorities, of his discovery of a heliocentric universe (Merriam-Paskow 1992: 42).

Además, toma sus decisiones de manera sencilla y sin desasosiego: "We never see Galileo agonize over his decisions. In fact he hardly seems to feel himself in much of a dilemma at all" (Merriam-Paskow 1992: 47). Por otra parte, como apunta C. Lorey, la ingenuidad egoísta lleva al protagonista al fracaso: "Beide Helden scheitern an einer wesenseigenen egoistischen Einfalt, die sowohl in ihrem Verhältnis zum Volk als auch in ihrem Umgang mit Frauen zutage tritt" ${ }^{22}$ (Lorey 1994: 253). La razón es otro aspecto imperante en la figura del protagonista de la obra. Esta, basada en la observación, condiciona su método científico y su visión del mundo. Sin embargo, en ocasiones peca de falta de razón. Cuando le advierten de que no vaya a Roma, él, aun sabiendo que sus teorías ya habían recibido el rechazo de las autoridades florentinas, decide ir para convencer a las personas que tienen la jurisdicción en la Iglesia. El resultado es la forzosa retractación de Galileo; si bien es cierto que continúa su labor científica con Discorsi.

\footnotetext{
${ }^{21}$ Andrea aclara al chico que recibe el libro que todavía no se sabe nada, esta situación solo es el comienzo: "Wir wissen bei weitem nicht genug, Giuseppe. Wir stehen wirklich erst am Beginn" (I3I).

22 La cita hace referencia a "Beide Helden" porque se trata de un estudio de los personajes de las obras Dantons Tod y Leben des Galilei.
} 
La entrega de Discorsi eclipsa el aspecto negativo causado por la retractación de Galileo: "Galilei's negative aspect was, however, overshadowed by his positive accomplishment - the delivery of his great work, the Discorsi" (Groseclose 1970: 367). A pesar de ello, esta retractación, aunque no se especifique en el texto dramático, pudo ser una maniobra de Galileo para ganar tiempo y poder propagar sus investigaciones: "Galilei recanted before the Inquisition only to gain time to

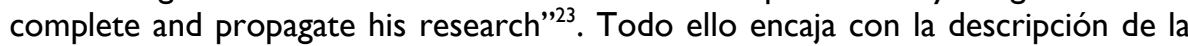
obra que hace C. Lorey. La verdad y la mentira jugarían un papel fundamental en la obra: "In beiden Stücken werden Lüge und Wahrheit zu Spielsteinen, die die Titelhelden und ihre Gegner nach persönlichem Nutzen und Belieben einsetzen"24 (Lorey 1994: 253).

\section{Símbolos}

Leben des Galilei contiene una serie de símbolos, distribuidos a lo largo de la obra, que se relacionan tanto con la responsabilidad de Galileo como con su método científico. Los que contienen un mayor valor literario y están directamente relacionados con los temas del presente estudio son la ceguera, la comida y la vestimenta.

\section{I. Ceguera y visión}

El protagonista basa sus teorías en sus observaciones; es decir, en su visión. Esta visión va empeorando con el transcurso de la obra, llegando al punto de impedirle reconocer a algún personaje. A. C. Fehn lo menciona como "Brecht's interweaving of plot and metaphor with respect to Galileo's blindness" (Fehn 1978: 30). Por tanto, se afirma que la metáfora de la ceguera de Galileo se entrelaza con la trama. Que base sus teorías en su visión ${ }^{25}$ significa por tanto que el científico iguala los conceptos sehen y beweisen: "he uses the word sehen almost synonymously with beweisen" (Fehn 1978: 27). Con relación a este hecho, Galileo asegura a su casera, la madre de Andrea, "Ich lehre ihn sehen" (I2).

La novena escena supone el comienzo de los problemas de visión de Galileo, cuando es incapaz de percibir a Ludovico, prometido de su hija Virginia. Esto limita

\footnotetext{
${ }^{23}$ Ibíd.

24 "beiden Stücken" hace referencia a las dos obras que trata el estudio; Dantons Tod y Leben des Galilei.

${ }^{25}$ L. D. Easton y K. H. Guddat consideran que la percepción por los sentidos es la base de la ciencia: "Sense perception must be the basis of all science. Science is only actual when it proceeds from sense perception in the twofold form of both sensuous awareness and sensuous need" (Easton / Guddat 1967: 3||$-3 \mid 2)$
} 
su razón, ya que esta anteriormente estaba conectada con la visión: "In the intervening scenes, images of vision help to define the limits of Galileo's reason" (Fehn 1978: 28). La limitación de la razón provoca que Galileo no sea consciente de los peligros:

Brecht emphasizes the metaphorical significance of the blindness by juxtaposing Galileo's refusal to admit that he may no longer be safe in Florence with a stage direction indicating his physical inability to recognize the "individual" who has been shadowing him (Fehn 1978: 30).

Sin embargo, a pesar de que el Galileo presente en el final de la obra tiene unos problemas de visión muy severos, consigue recopilar todas sus investigaciones en Discorsi.

Finalmente cabe destacar la manera en la que Galileo reta al poder establecido. La lucha entre la Iglesia y Galileo imperante en la obra no es más que una batalla que libra el mencionado científico mediante la autoridad y la razón que le confieren sus ojos:

Galileo challenges "the Ancients," by recognizing no authority other than his own eyes. He thereby performs, what might be called a castration of the eyes of God as privileged looker and, by extension, a castration of the eyes of His ministers, the witnesses, interpreters and executors of God's view on earth (Merriam-Paskow 1992: 51).

El desplazamiento que hace Galileo de la visión de Dios hacia un segundo plano para dar ese lugar privilegiado a la visión del ser humano provoca que la Iglesia se vea amenazada y rechace las teorías.

\subsection{Comida}

La comida es otro símbolo que juega un papel fundamental en la obra, puesto que se puede relacionar con sucesos que atañen a la responsabilidad y al método científico de Galileo.

En primer lugar destaca el hecho de que sus deseos de investigar estén vinculados en la obra con la comida: "His interest in investigating is linked rather, and most explicitily, with food, his pleasure of discovery with oral gratification. The play, in fact, begins and ends with food" (Merriam-Paskow 1992: 44). Además, como muestra la afirmación de Merriam-Paskow y la propia obra de Brecht, el drama comienza y termina con Galileo y Andrea tratando temas científicos y con alimentos; leche al principio y ganso al final. Esto se refleja en la visita que hace Andrea a Galileo cuando este se ha retractado públicamente debido a la presión de la Iglesia. Galileo hace entrega de Discorsi a Andrea y le manifiesta que, a pesar de estar controlado por la Inquisición, ha recopilado sus teorías. Galileo sentía la 
necesidad de hacerlo, ya que investigar se había convertido en algo instintivo: "as pleasurable and as instinctive as eating, ceases to be an achievement and becomes a vice" (Fehn 1978: 31).

Por último, destaca la relación que establece el Papa entre el deseo de investigar de Galileo y su deseo por el vino: "Zu einem alten Wein oder einem neuen Gedanken könnte er nicht nein sagen" (108). "And indeed, the only times Galileo "declares his love" in the play, it is his love for truth and his love of a particularly tasty wine that he drinks on the occasion of his decision to investigate the sun spots" (Merriam-Paskow 1992: 44-45).

\subsection{Vestimenta}

El símbolo de la vestimenta aparece en dos partes del drama. El comienzo de este presenta a Galileo con el torso desnudo, limpiándose. Posteriormente se viste y explica a Andrea sus teorías haciendo uso de algunos objetos. Por su parte, el Papa juzga a Galileo mientras se está vistiendo. Además, emplea la tecnología para amedrentar al protagonista: "In Scene I Galileo appears naked from the waist up. ... He demonstrates to Andrea how the earth revolves around the sun ... In Scene 12 Pope Urban VIII ... is being dressed during an audience ... he decides that Galileo should be shown the instruments of torture" (Sohlich 1993: 55). La vestimenta en este caso se relaciona con el uso que hace Galileo de la razón y la tecnología para exponer a su alumno sus investigaciones y lo que él considera como verdad y el uso que hace la Iglesia de la tecnología para reprimir las teorías que van en contra de sus intereses.

\section{Conclusiones}

Los científicos cumplen un papel fundamental. Se afirma con frecuencia que el ser humano es curioso por naturaleza. Esta necesidad de saber es satisfecha por los científicos, los cuales mediante sus investigaciones, siguiendo un determinado método científico, acercan a los demás el nuevo conocimiento. Pero este conocimiento debe ajustarse a la realidad, sin esconder información atendiendo a determinados intereses. Cuando esto ocurre, se está faltando a la ciencia.

Galileo, siempre tomando el camino hedonista, se encuentra con que debe abandonar sus investigaciones de manera forzosa, debido a que estas van en contra de una institución que ostentaba el poder en Italia, la Iglesia. Sin embargo, aunque renuncie a ello, su arrepentimiento le hace recopilar sus teorías en un libro y entregárselo a su alumno para que lo lleve fuera del país. El protagonista, pese a 
haber llevado a cabo esta acción, se arrepiente de haberse rendido, puesto que considera que ha fallado a la ciencia.

Mediante la historia, Bertolt Brecht consigue crear un drama perteneciente al teatro épico. La finalidad de este teatro no era otra que conseguir que los espectadores reflexionaran evitando la catarsis propia del teatro aristotélico. En 1939 estalló la Segunda Guerra Mundial y muchos escritores vieron como sus obras se censuraban por ir en contra de las ideas de los que controlaban el estado. Por tanto, la obra tendría como objetivo conseguir que el espectador relacionase ambas épocas y sucesos, de manera que actuara para cambiar la situación.

\section{Referencias bibliográficas}

Bibliografia primaria

Brecht, B. (20I7), Leben des Galilei. Berlín: Suhrkamp Verlag.

Bibliografia secundaria

Braga, M. A. B. / Medina, M. N. (20I0). O teatro como ferramenta de aprendizagem da física e de problematização da natureza da ciencia. Caderno brasileiro de ensino de física, 2, 27: 313-333.

Brecht, B. (1967). Schriften zur Literatur und Kunst. Berlín: Suhrkamp Verlag.

Cohen, M. A. (1970). History and Moral in Brecht's 'The Life of Galileo'. Contemporary Literature, I, I I: 80-97.

Dorsey, J. T. (1987). The Responsibility of the Scientist in Atomic Bomb Literature. Comparative Literature Studies, 3, 24: 277-290.

Easton, L. D. / Guddat, K. H. (1967). Writings of the young Marx on philosophy and society. Garden City, NY: Doubleday.

Edsall, J. T. (198I). Two Aspects of Scientific Responsibility. Science, 4490, 2 I2: I I14.

Fehn, A. C. (1978). Vision and Blindness in Brecht's 'Leben des Galilei'. Germanic Review, 53 (I): 27-34.

Gimenez, H. / Rinaldi, C. (20I5). Teatro científico: $O$ ensino de ciências em quatro atos. Revista Internacional de Apoyo a la Inclusión, Logopedia, Sociedad y Multiculturalidad, 4(I): 70-84.

Groseclose, J. S. (1970). Scene Twelve of Bertolt Brecht's 'Galilei': A Structural Study. Monatshefte, 62 (4): 367-382.

Lorey, C. (1994). Glaube und Zweifel, Lüge und Wahrheit, Genialität und Einfalt. Georg Büchners 'Dantons Tod' und Bertolt Brechts 'Leben des Galilei'. Deutsche Vierteljahrsschrift für Literaturwissenschaft und Geistesgeschichte 68(2): 25।-277.

Merriam-Paskow, J. (1992). Brecht's 'Leben des Galilei': The Modern Scientist as Voyeur. Modern Language Studies, 4, 22, 42-56. 
Paulsell, P. R. (1988). Brecht's treatment of the Scientific Method in His 'Leben des Galilei'. German Studies Review, 2, I I, 267-284.

Riemen, R. (20II), "Hoe inspannend het is om een Boze te zijn”, Theater Schrift Lucifer, 12, 106-123.

Sohlich, W. (1993), "The Dialectic of Mimesis and Representation in Brecht's 'Life of Galileo'”, Theatre Journal, I, 45, 49-64.

Suvin, D. (1990), “Brecht's 'Life of Galileo': Scientistic Extrapolation or Analogy of the Knower?", Forum Modernes Theater, 2, 5, II9-I38.

Taylor, F. S. (1938), Galileo and the Freedom of Thought. London: Watts.

Weinberg, A. M. (1978), "The obligations of citizenship in the republic of science", Minerva, I, 16, I-3.

Zanarini, D. (20I7), "La obra 'Vida de Galileo' de Bertolt Brecht como recurso didáctico para una enseñanza contextualizada de la física”, Revista de Enseñanza de la Física, Extra, 29, 49I-498. 
